\title{
A combined flood risk assessment with SWMM hydrological modelling and AHP based on multi-criteria decision-making
}

\author{
Yanping $\mathrm{Li}^{\mathrm{a}, \mathrm{b}}$, Tao Wang a,b, *, Xiwei Wang ${ }^{\mathrm{a}, \mathrm{b}}$, Xiaojuan Li ${ }^{\mathrm{a}, \mathrm{b}}$ \\ ${ }^{a}$ MOE Lab of 3D Spatial Data Acquisition and Application, Capital Normal University, Beijing, 100048, China, \\ 2190902188@cnu.edu.cn,wangt@cnu.edu.cn,2200902108@cnu.edu.cn, lixiaojuan@cnu.edu.cn \\ ${ }^{b}$ College of Resource Environment and Tourism, Capital Normal University, Beijing, 100048, China \\ * Corresponding author
}

Keywords: urban flood disaster, risk evaluation, SWMM, AHP, Visualization

\begin{abstract}
:
In the context of global warming and climate changes coupled with urbanization, floods due to intensive precipitation poses huge risks to urban regions where high density population and economic indications locate.

Flood modelling can typically be implemented in Distributed hydrological modelling and AHP based evaluation. Distributed hydrological modelling can simulate surface runoff and map flooding areas. Storm water management module (SWMM), developed by the US Environmental Protection Agency (EPA), is a dynamic hydrology-hydraulic water quality simulation model and used to plan, analyse and design drainage systems in urban areas. This hydrological modelling was developed to reduce runoff through infiltration and retention, and help to reduce discharges that cause impairment of waterbodies. Using natural and social factors inducing floods, the AHP based method analyses basic parameters in one mathematical model and evaluate flood risk of a specific region.
\end{abstract}

These two models evaluate flood from different perspectives. Both have their special advantages. A combined approach can make the two models complement to each other. SWMM hydrological modelling can simulate the flood process and obtain the waterlogging information directly. And the AHP based method combines influencing factors to evaluate the risk of flood disaster. Therefore the results of disaster factors evaluation and process simulation evaluation complement and verify each other.

This study selects an area of Liangxiang Town and Changyang Town, which is located in the southwest of Beijing, covering an area of 220 square kilometres. Due to fast urbanization in recent years, transformation of land cover from rural settings to impervious surface has been happening continuously. And as one of the 14 satellite towns of Beijing, this region attracts intensive investment and population. Southeast to this area is plain facing Bohai sea and northwest is mountainous. And during monsoon season each summer heavy rainfalls generate flood risks in this region.

To implement the two models, the authors collected data including remote sensing imagery, DEM, meteorological records, socio-economic statistical data, urban pipe network data and historical disaster data. Land cover data area extected from satellite images. The rainfall and storm patterns are obtained by analyzing the meteorological data.

SWMM hydrological model simulates the urban flood process with inputs including rainfall data and the waterlogging nodes. Drainage information can be obtained through simulating the urban pipeline drainage system. And surface runoff with different levels of rainfall will be simulated. AHP based method combines the topographical, hydrological, land cover and precipitation data. And flood risk map will be produced. Results from these two approaches will be compared quantitatively and visually. Preliminary results will be presented during conference.

The results of this study in a cross validated model can better serve risk resilient urban planning and help city managers better prepared in the area like transportation and insurance. 